\title{
Resistência compressiva da resina composta na face proximal de cavidades classe II em diferentes profundidades: estudo in vitro
}

\section{Composite resin compressive strength in the proximal surface of class II cavities at different depths: an in vitro study}

Laryssa Signor

Angela Isabel Dullius*

Gislaine Rosa Biacchi*

\section{Resumo}

Apesar do notável aumento, nos últimos anos, de restaurações posteriores com resinas compostas, ainda existem dificuldades quanto à técnica restauradora em se tratando de cavidades com envolvimento da face proximal. Considera-se que a intensidade de luz que atinge os incrementos da resina na face cervical do preparo é menor do que a intensidade próxima à superfície, podendo comprometer as propriedades mecânicas e o sucesso da restauração. Objetivo: o objetivo deste trabalho foi investigar a resistência compressiva dos incrementos de resina composta de uso direto na profundidade da caixa proximal de restaurações classe Il em diferentes extensões ocluso-cervicais. Materiais e método: foram obtidos 40 espécimes (blocos) de resina composta, com $2 \mathrm{~mm}$ de dimensão ocluso-cervical, confeccionados a partir de restaurações classe II em três molares inferiores de manequim (Prodens), com dimensões de $4 \mathrm{~mm}$ vestíbulo-lingual, $2 \mathrm{~mm}$ mésio-distal e profundidades ocluso-cervicais de $2 \mathrm{~mm}$ (G1 e G2), $4 \mathrm{~mm}(G 3)$ e $6 \mathrm{~mm}(G 4)$, formando 4 grupos $(n=10)$. Para G1 (grupo controle), foram utilizadas a resina autoativada (Alpha Plast) e demais grupos de resina fotoativada (Z100, 3M ESPE), todas com espessura de $2 \mathrm{~mm}$. Posteriormente, os espécimes foram armazenados em soro fisiológico durante 30 dias e, em seguida, submetidos ao teste de resistência à compressão em máquina universal de ensaios EMIC DL-2000 - composta por um dispositivo metálico de base plana de $15 \mathrm{~mm}$, com célula de carga de $490 \mathrm{~N}$ e velocidade de $0,5 \mathrm{~mm} / \mathrm{min}$ -, aplicado sobre a face oclusal dos espécimes até a fratura. Os resultados foram analisados pelo teste Ano- va. Resultados: as médias dos valores de resistência à fratura (MPa) foram de: 52,86; 48,55; 44,89; 42,67 para G1, G2, G3 e G4, respectivamente. Conclusão: não houve diferença significativa $(p>0,05)$ na resistência à compressão entre incrementos de resina composta inseridos na base da face proximal de preparos do tipo classe II em diferentes profundidades, utilizando-se o protocolo adotado.

Palavras-chave: Restauração dentária permanente. Resina. Materiais dentários.

\section{Introdução}

As resinas compostas, inicialmente elaboradas para confecção de restaurações estéticas em dentes anteriores, passaram a ser utilizadas também em dentes posteriores ${ }^{1,2}$. Contudo, algumas propriedades inerentes a esse material, como a contração de polimerização e o coeficiente de expansão térmica linear diferente da estrutura dental, podem acarretar em falhas nas restaurações ${ }^{3}$.

Apesar do avanço no que se refere às propriedades das resinas compostas, ainda existem dificuldades quanto à técnica de restauração, principalmente quando se trata da adaptação do material à parede cervical da caixa proximal em restaurações classe II, em que o acesso da luz para a polimerização é mais difícil ${ }^{4}$.

** Professora Adjunta do Curso de Odontologia da Universidade Federal de Santa Maria (UFSM), Santa Maria, RS, Brasil. 
Para que os materiais restauradores estéticos tenham adequadas propriedades mecânicas e físicas, faz-se necessário uma polimerização eficiente ${ }^{5}$. Caso contrário, poderá ocorrer maior contração de polimerização ${ }^{6,7}$, instabilidade de $\operatorname{cor}^{8}$, agressão pulpar $^{9}$, maior risco de cárie secundária ${ }^{10,11}$ e diminuição da dureza ${ }^{12}$.

Considerando que, com a face cervical da caixa proximal de restaurações classe II, a intensidade de luz que atinge os incrementos da resina deva ser consideravelmente menor do que a intensidade próxima à superfície, o grau de conversão dos monômeros em polímeros também sofre um decréscimo ${ }^{13}$.

Com a finalidade de controlar a contração de polimerização, Lutz et al. ${ }^{14}$ (1986) desenvolveram a técnica incremental, para restaurações classe II, na qual a resina é inserida em várias camadas. Pollack $^{15}$ (1988) sugere que os incrementos sejam inseridos de forma oblíqua, pois isso propicia uma maior superfície livre para liberação do estresse, o que resulta na diminuição do fator $\mathrm{C}$, fator da configuração cavitária. Esses incrementos devem ser também de no máximo $2 \mathrm{~mm}$, pois, quando de maior espessura, não permitem uma polimerização uniforme e máxima ${ }^{16}$. Por meio da redução do volume de resina composta a ser polimerizada de cada vez, a tensão marginal torna-se menor ${ }^{14,15}$.

A propriedade mecânica de resistência à compressão demonstra a capacidade do compósito em suportar estresses verticais, pois as forças transmitidas às restaurações durante $o$ ato mastigatório podem desencadear fraturas do compósito e/ou do elemento dental ${ }^{17,18,19}$.

Sabendo que o grau de conversão da resina está diretamente ligado às propriedades mecânicas do material e, consequentemente, ao seu desempenho clínico, este trabalho tem a finalidade de investigar a resistência compressiva dos incrementos de resina composta fotoativados na base da caixa proximal de restaurações classe II em diferentes profundidades, comparando com uso de resinas autoativadas que independem de luminosidade para a polimerização, segundo o protocolo restaurador adotado.

\section{Materiais e método}

Foram confeccionados 40 espécimes de resina composta obtidos a partir de restaurações classe II em dentes molares. Para isso, prepararam-se cavidades nas faces distais de três primeiros molares inferiores (dente 36) de manequim (Prodens, São Gonçalo, RJ, Brasil), com dimensões de $4 \mathrm{~mm}$ vestíbulo-lingual, $2 \mathrm{~mm}$ mésio-distal e profundidades ocluso-cervicais de $2 \mathrm{~mm}, 4 \mathrm{~mm}$ e $6 \mathrm{~mm}$, medidas a partir do sulco central da face oclusal. As dimensões do preparo foram determinadas com o auxílio de uma espátula de madeira adaptada para este fim, com as marcações das profundidades mensuradas com o auxílio de um paquímetro digital (Kingtools,
São Paulo, SP, Brasil). O preparo foi iniciado com broca diamantada cilíndrica 1093 em alta rotação (KG Sorensen, Barueri, SP, Brasil) e complementado com broca diamantada tronco-cônica 6847 (Komet, Santo André, SP, Brasil) de haste longa em baixa rotação. Para a finalização dos preparos, utilizaram-se cinzel número 2 (Neumar, Pirituba, SP, Brasil) e ponta abrasiva de silicone (Optimize, TDV, Pomerode, SC, Brasil). Os dentes foram adaptados ao manequim articulado (Prodens, São Gonçalo, RJ, Brasil) no encaixe destinado ao elemento dentário 36, mantendo-se, na posição, o segundo molar inferior. Foi adaptada uma matriz metálica de $7 \mathrm{~mm}$, estabilizando-a com uma cunha de madeira e um anel Unimatrix (Optimize, TDV, Pomerode, SC, Brasil) entre a face proximal a ser restaurada e 0 dente adjacente (Figura 1).

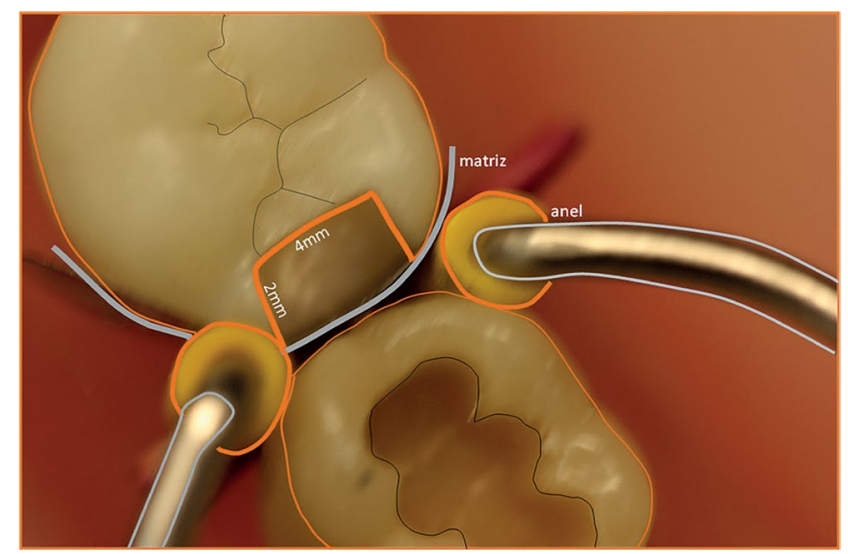

Figura 1 - Dente 36 em posição e adaptado para a confecção dos espécimes

Fonte: dos autores.

Uma camada fina de isolante para resina (Cel-Lac, SSWhite, Rio de Janeiro, RJ, Brasil) foi aplicada com pincel (KG Brush, Cotia, SP, Brasil) e seca naturalmente antes da confecção dos espécimes para facilitar a remoção. Os espécimes de resina composta fotoativada foram confeccionados na caixa proximal dos dentes preparados, envolvendo as faces cervicais, mésio-distais e axiais, com altura de $2 \mathrm{~mm}$ pela inserção de três incrementos do material, conforme preconizado por Pollack ${ }^{15}$ (1988). Os espécimes com resina composta autoativada foram confeccionados com um só incremento. Quatro grupos foram obtidos com 10 espécimes $(\mathrm{n}=10)$ cada em forma de blocos de resina, nas dimensões médias de: $2 \mathrm{~mm}$ de espessura, $2 \mathrm{~mm}$ de largura disto-axial e 2 de largura cervico-oclusal. O Grupo 1 (G1), grupo controle, teve seus espécimes confeccionados com resina composta autoativada Alpha Plast (Nova DFL, Jacarepaguá, RJ, Brasil), obtidos a partir dos dentes, com preparos cavitários de $2 \mathrm{~mm}$ de profundidade ocluso-cervical, com inserção em bloco único do material após manipulação conforme instruções do fabricante. Para o Grupo 2 (G2), os espécimes de resina composta fotoativada foram obtidos a partir dos mesmos dentes preparados com $2 \mathrm{~mm}$ de profun- 
didade. Para o Grupo 3 (G3), os espécimes foram obtidos a partir de dentes preparados com profundidade ocluso-cervical de 4mm. E, no Grupo 4 (G4), a partir de dentes preparados com profundidade ocluso-cervical de $6 \mathrm{~mm}$. O primeiro incremento foi inserido sobre a metade da parede gengival e toda a parede vestibular, o segundo foi inserido da mesma forma sobre a parede lingual, e o terceiro incremento preencheu o espaço central, fechando a caixa proximal (Figura 2).

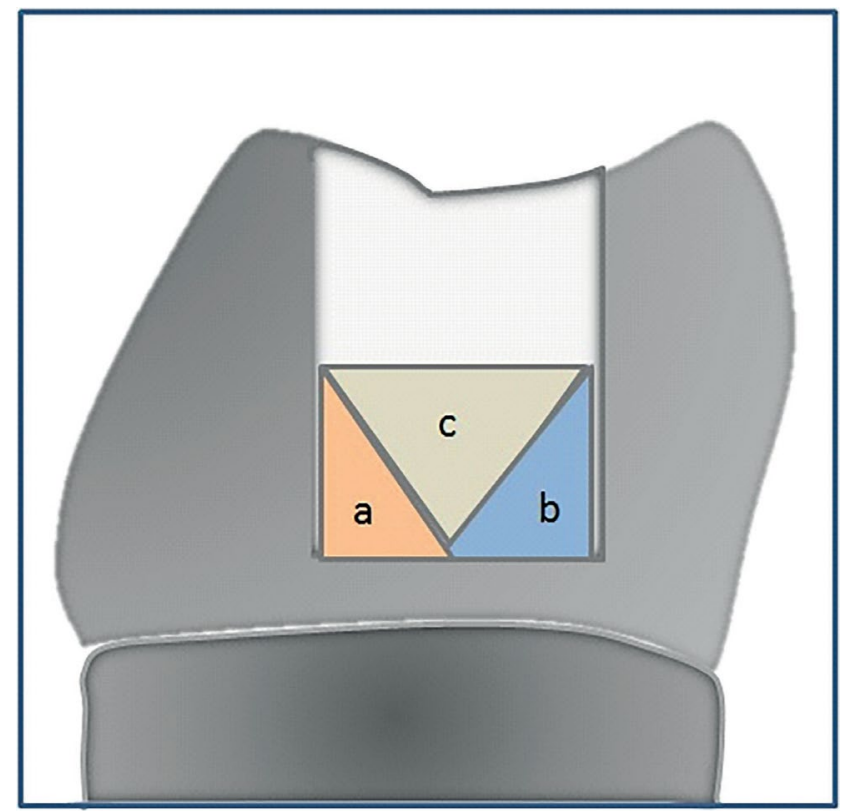

Figura 2 - Desenho esquemático dos incrementos de resina em dente preparado na profundidade de $4 \mathrm{~mm}$ : A (vestíbulo/gengival); $B$ (linguo-gengival); e $C$ (incremento central).

Fonte: dos autores.

Para a confecção dos espécimes destes grupos, utilizou-se a resina composta Z100 (3M do Brasil Ltda, Sumaré, SP, Brasil) na cor A2, inserida com auxílio de espátulas de titânio número 1 (1-SD1, Millennium, São Caetano do Sul, SP, Brasil) e número 6 (Indusbello, Londrina, PR, Brasil) até o limite de $2 \mathrm{~mm}$, previamente definido. A fotoativação foi realizada com aparelho de LED (Schuster Emitter C, Santa Maria, RS, Brasil) e intensidade de luz média de $900 \mathrm{~mW} / \mathrm{cm}^{2}$, por 40 segundos, após a inserção de cada incremento, com a ponta aplicadora posicionada na face oclusal do preparo. Optou-se pelo tempo de 40 segundos para aumentar a exposição à luz e converter o maior número de monômeros em polímeros, uma vez que o afastamento, pela profundidade cavitária, da luz do fotopolimerizador poderia prejudicar a polimerização do material ${ }^{20}$. Antes da confecção de cada espécime, foi registrada a intensidade do fotopolimerizador com o auxílio de um radiômetro digital (Ecel RD-7, Ribeirão Preto, SP, Brasil). Depois a confecção, o espécime foi removido com o auxílio de uma espátula 3S (Millennium, São Caetano do Sul, SP, Brasil), e as irregularidades dos blocos (espículas) de resina composta foram removidas com lixas abrasivas de granulação 120, para obter a estabilidade necessária ao teste de compressão. Posteriormente os espécimes foram armazenados em água destilada, em recipientes do tipo Eppendorf, em temperatura ambiente. Na sequência, sobre a face oclusal dos espécimes, utilizando-se um dispositivo metálico de base plana de $15 \mathrm{~mm}$, aplicou-se força de compressão utilizando-se a máquina universal de ensaios (EMIC DL-2000, São José dos Pinhais, PR, Brasil), com célula de carga de $490 \mathrm{~N}$ e velocidade de $0,5 \mathrm{~mm} / \mathrm{min}$, até a fratura. Os resultados foram registrados, e a resistência à compressão em MPa foi calculada a partir da fórmula: $\mathrm{Mpa}=$ Força $(\mathrm{KgF}) /$ área $\left(\mathrm{cm}^{2}\right)$.

\section{Análise estatística}

A estatística descritiva e a normalidade dos dados foram constatadas pelo teste de Shapiro Wilk ( $p>0,05)$, a partir do software SPSS v 17.0. Os valores obtidos no teste de resistência foram submetidos à Análise de Variância (Anova), a fim de detectar as variações significantes entre os grupos, considerando o nível de significância de $5 \%$.

\section{Resultados}

Os valores médios de resistência à compressão para os diferentes grupos e os respectivos desvios padrão estão apresentados na Tabela 1.

Tabela 1 - Valores médios de resistência à fratura (MPa), desvio padrão $(S D)$, valores mínimos e máximos e análise estatística

\begin{tabular}{l|c|c}
\hline \multicolumn{1}{c|}{ Grupo } & Média $( \pm$ SD)* & Valor Mínimo - Valor Máximo \\
\hline G1 (controle) & 52,86 A $(11,71)$ & $34,51-70,09$ \\
G2 (2mm) & 48,55 A $(9,84)$ & $37,06-65,22$ \\
G3 (4mm) & 44,89 A $(9,55)$ & $32,13-53,12$ \\
G4 (6mm) & 42,67 A $(7,74)$ & $28,04-54,14$ \\
\hline
\end{tabular}

dos autores.

* Médias seguidas de letras iguais não têm diferença estatística $(p \geq 0,05)$.

É possível verificar, nos resultados encontrados, que a resistência compressiva da resina composta diminuiu com a distância da fonte luminosa, porém, sem significância estatística, considerando o protocolo adotado na inserção dos incrementos do material, bem como o tempo de fotoativação de cada incremento. Não houve diferença estatística entre o grupo controle e os grupos experimentais.

\section{Discussão}

Restaurações posteriores de resina composta são procedimentos largamente utilizados na clínica odontológica. Em se tratando de restaurações classe II que envolvem as faces proximais do dente, seja 
distal, mesial ou ambos, a confecção deste procedimento torna-se um desafio clínico, visto que a excelência funcional da restauração está diretamente ligada à adequada adesão às paredes dentárias e à capacidade de ampla polimerização do material.

Sendo as faces proximais profundas, a distância entre a ponta do fotoativador e o adesivo e, posteriormente, a resina composta é ampliada, prejudicando a polimerização dos materiais e, consequentemente, suas propriedades físicas e mecânicas ${ }^{5}$.

Este estudo buscou avaliar as consequências do aumento da profundidade de um preparo cavitário na face distal de um dente sob o ponto de vista da resistência mecânica do material, propriedade necessária a uma ideal função mastigatória ${ }^{17,18}$.

Artifícios técnicos na confecção de restaurações posteriores classe II, ou mesmo classe I profunda, devem ser adotados para que se obtenha um resultado condizente com as necessidades restauradoras em dentes posteriores: função, longevidade e estética. Ao adotar-se a estratégia de inserção do material em pequenos incrementos, como a desenvolvida por Pollack ${ }^{15}$ (1988), aliada ao aumento da radiação de luz sobre cada porção, o resultado de polimerização pode ser efetivo, e restaurações, especialmente classe II, de resina composta em dentes posteriores podem ser uma possibilidade apropriada, com melhora nas propriedades mecânicas. No entanto, a fonte de luz deve ter uma intensidade razoável. No presente trabalho, utilizou-se um fotoativador com irradiância de $900 \mathrm{~mW} / \mathrm{cm}^{2}$, o que conferiu uma adequada polimerização do material restaurador, mesmo com o aumento da profundidade, e, consequentemente, uma resistência mecânica compressiva satisfatória. Conforme esperado, os resultados do teste à compressão, nos diferentes grupos, demonstraram um decréscimo da resistência com o aumento da profundidade do preparo, porém não estatisticamente significante.

Dessa forma, pode-se levantar a possibilidade de que fontes de luz com menor irradiância poderão acarretar em um material menos polimerizado, sujeito a menor resistência compressiva. Além disso, o tempo de polimerização foi ampliado para 40 segundos, o dobro de tempo preconizado pelo fabricante para cada incremento, visto que o tempo de polimerização influencia na dureza do material. Fato observado em um estudo realizado por Brosh et al. ${ }^{21}$ (1997), no qual fotoativaram-se resinas compostas com 20, 40, 60 e 180s.

Ao comparar a resina fotoativada com a resina autoativada, buscou-se avaliar se maiores resistências à compressão seriam alcançadas quando são dispensadas as condições de luminosidade e as inserções por incremento, necessárias ao primeiro material. Embora tenham sido obtidos resultados médios superiores com a resina composta autoativada, observou-se que estes não foram estatisticamente significantes. Porém, vale lembrar que, com as resinas fotoativadas, utilizou-se um protocolo de inserções sequenciais ${ }^{15}$ com tempos de ativação de 40 segundos para cada um dos três incrementos, tempo superior ao sugerido pelo fabricante. São necessários estudos com diferentes protocolos e tempos de ativação, utilizando-se a metodologia descrita com cavidades preparadas em dentes naturais ou artificiais, para avaliar se resultados semelhantes podem ser alcançados.

Como vários estudos demonstram a dificuldade de polimerização de resinas compostas com o aumento da distância da luz do aparelho fotoativador, pode-se considerar a utilização de resinas autoativadas, em especial como base de restaurações classe II profundas. Dessa forma, seria atenuado o problema de polimerização nas áreas em que a profundidade da cavidade impede ou reduz a incidência da luz sobre o material, influenciando na sua resistência. Resinas do tipo Bulk Fill estão sendo utilizadas para obterem-se melhores resultados nas restaurações de classe II, contudo, também são materiais fotoativados dependentes de uma alta intensidade de luz dos aparelhos fotopolimerizadores ${ }^{22}$.

\section{Conclusão}

Considerando as limitações deste estudo in vitro, pode-se concluir que não houve diferença significativa na resistência à compressão entre blocos de resina composta inseridas na base da face distal de preparos do tipo II em diferentes profundidades, quando adotam-se técnica incremental, fonte de luz de alta irradiância e tempo de fotoativação aumentado.

\section{Abstract}

Despite the remarkable increase of posterior restorations with composite resins over the last years, there are still difficulties regarding the restorative technique when dealing with cavities involving the proximal surface. It is considered that the light intensity that reaches resin increments in the cervical aspect of the preparation is lower than the intensity close to the surface, which may compromise the mechanical properties and the success of the restoration. Objective: to investigate the compressive strength of composite resin increments for direct use in the depth of the proximal box of class II restorations in different occlusal-cervical extensions. Methods: 40 specimens (blocks) of composite resin were obtained. They had $2 \mathrm{~mm}$ of occlusal-cervical dimension and were made from class II restorations in three lower molars of a dental manikin (Prodens) with $4 \mathrm{~mm}$ buccolingual and $2 \mathrm{~mm}$ mesiodistal dimensions, and occlusal-cervical depths of $2 \mathrm{~mm}$ (G1 and G2), 4mm (G3), and $6 \mathrm{~mm}(\mathrm{G} 4)$, forming 4 groups $(n=10)$. The control group (G1) used the self-activated resin (Alpha Plast) and other photoactivated resin groups (Z100, 3M ESPE), which were all $2 \mathrm{~mm}$ thick. Next, the specimens were stored in saline solution for 30 days and then subjected to the compressive strength test in a universal test machine EMIC DL-2000, consisting of a $15 \mathrm{~mm}$ flat base metal 
device with a load cell of $490 \mathrm{~N}$ and velocity of $0.5 \mathrm{~mm} /$ min applied to the occlusal surface of the specimens until fracture. The results were analyzed by ANOVA. Results: the mean fracture resistance $(\mathrm{MPa})$ values were 52.86, 48.55, 44.89, and 42.67, for G1, G2, G3, and G4, respectively. Conclusion: there was no significant difference $(p>0.05)$ in the compressive strength among composite resin increments inserted in the base of the proximal surface of class II preparations at different depths using the protocol adopted.

Keywords: Permanent dental restoration. Resin. Dental materials.

\section{Referências}

1. Kurokawa H, Takamizawa T, Rikuta A, Tsubota K, Miyazaki M. Three-year clinical evaluation of posterior composite restorations placed with a single-step self-etch adhesive. J Oral Sci 2015; 57(2):101-8.

2. Bohaty BS, Ye Q, Misra A, Sene F, Spencer P. Posterior composite restoration update: focus on factors influencing form and function. Clin Cosmet Investig Dent 2013; 15(5):33-42

3. Casanova RC, Amaral CM, Marchi GM, Pimenta LAF, Costa JF. Influência das técnicas de inserção de resina composta condensável sobre a infiltração marginal. Cienc Odontol Bras 2002; 5(3):62-9.

4. Chain MC. Unidades fotoativadoras de luz visível: foto-polimerizadores. In: Baratieri LN. Estética: restaurações adesivas diretas em dentes anteriores fraturados. 2. ed. São Paulo: Santos Livraria Editora; 1998. p. 117-33.

5. Davidson-Kaban SS, Davidson CL, Feilzer AJ, de Gee AJ, Erdilek N. The effect of curing light variations on bulk curing and wall-to-wall quality of two types and various shades of resin composites. Dent Mater 1997; 13(6):344-52.

6. Benetti AR, Havndrup-Pedersen C, Honoré D, Pedersen MK, Pallesen U. Bulk-fill resin composites: polymerization contraction, depth of cure, and gapformation. Oper Dent 2015; 40(2):190-200.

7. Dewaele M, Asmussen E, Peutzfeldt A, Munksgaard EC, Benetti AR, Finné G et al. Influence of curing protocol on selected properties of light-curing polymers: degree of conversion, volume contraction, elastic modulus, and glass transition temperature. Dent Mater 2009; 25(12):1576-84.

8. Karaman E, Tuncer D, Firat E, Ozdemir OS, Karahan S. Influence of different staining beverages on color stability, surface roughness and microhardness of silorane and methacrylate-based composite resins. J Contemp Dent Pract 2014; 15(3):319-25.

9. Tamilselvam S, Divyanand MJ, Neelakantan P. Biocompatibility of a conventional glass ionomer, ceramic reinforced glass ionomer, giomerand resin compositeto fibroblasts: in vitro study. J Clin Pediatr Dent 2013; 37(4):403-6.

10. Lai GY, Zhu LK, Li MY, Wang J. An in vitro study on the secondary caries-prevention properties of three restorative materials. J Prosthet Dent 2013; 110(5):363-8.

11. Kopperud SE, Tveit AB, Gaarden T, Sandvik L, Espelid I. Longevity of posterior dental restorations and reasons for failure. Eur J Oral Sci 2012; 120(6):539-48.

12. Lima AF, de Andrade KM, da Cruz Alves LE, Soares GP, Marchi GM, Aguiar FH et al. Influence of light source and extended time of curing on microhardness and degree of conversion of different regions of a nanofilled composite resin. Eur J Dent 2012; 6(2):153-7.
13. Mousavinasab SM, Barekatain M, Sadeghi E, Nourbakhshian F, Davoudi A. Evaluation of light curing distance and mylar strips color on surface hardness of two different dental composite resins. Open Dent J 2014; 8(8):144-7.

14. Lutz F, Krejci I, Oldenburg TR. Elimination of polymerization stresses at the margins of posterior composite resin restorations: a new restorative technique. Quintessence Int $1986 ; 17(12): 777-84$.

15. Pollack BF. Class II composites: 1987 thoughts and techniques. Oral Health 1988; 78(4):23-5.

16. Aguiar FH, Andrade KR, Leite Lima DA, Ambrosano GM, Lovadino JR. Influence of light curing and sample thickness on microhardness of a composite resin. Clin Cosmet Investig Dent 2009; 6(1):21-5

17. Batalha-Silva S, de Andrada MA, Maia HP, Magne P. Fatigue resistance and crack propensity of large MOD composite resin restorations: direct versus CAD/CAM inlays. Dent Mater 2013; 29(3):324-31.

18. van Dijken JW, Pallesen U. Randomized 3-year clinical evaluation of Class I and II posterior resin restorations placed with a bulk-fill resin composite and a one-step self-etching adhesive. J Adhes Dent 2015;17(1):81-8.

19. Opdam NJ, van de Sande FH, Bronkhorst E, Cenci MS, Bottenberg P, Pallesen U et al. Longevity of posterior composite restorations: a systematic review and meta-analysis. J Dent Res 2014; 93(10):943-9.

20. Borges A, Pitta-Lopes J, Portugal J. Influência do tempo de exposição e distância à luz na capacidade de fotopolimerização de compósitos. Revista Portuguesa de Estomatologia, Rev Port Estomatol Med Dent Cir Maxilofac jul./set. 2015; 56(3):166-72.

21. Brosh T, Baharau H, Gross O, Laufer BZ. The influence of surface loading and irradiation time during curing on mechanical properties of a composite. J Prosthet Dent 1997; 77(6):573-7.

22. Alkhudhairy F. The effect of curing intensity on mechanical propoerties of different bulk-fill composite resins. Clin Cosmet Investig Dent 2017; 23(9):1-6.

\section{Endereço para correspondência:}

Gislaine Rosa Biacchi

Rua Conrado Hoffman, 345, ap. 502

97060-140, Santa Maria, RS, Brasil

Telefones: (55) 3304-1209 e (55) 99914-5517

E-mail: gisarb@gmail.com

Recebido: 15/05/2017. Aceito: 30/06/2017. 